\title{
Paulina TARASEWICZ
}

Université de Gdańsk

\section{Bataille funèbre : \\ Lord Auch versus le cadavre divin}

\author{
Spectre en larmes \\ ô Dieu mort \\ cil cave \\ moustache humide \\ dent unique \\ ô Dieu mort \\ ô Dieu mort \\ Moi \\ je te poursuivais \\ de haine \\ insondable \\ et je mourais de haine \\ comme un nuage \\ se défait. \\ G. Bataille ${ }^{1}$
}

Du côté de Nietzsche

ue Nietzsche soit un des repères les plus constants dans la pensée et l'œuvre de Bataille est indéniable. Les faits sont largement connus : la lecture de Par-delà le bien et le mal provoque en lui une sorte de révélation, elle est un des facteurs majeurs de sa perte de foi ; Contre-Attaque, mouvement antifasciste qu'il anime avec André Breton en 1935, est conçu sous l'inspiration nietzschéenne, de même qu'Acéphale, la revue dont le deuxième numéro est entièrement consacré au philosophe allemand. Parmi les conceptions nietzschéennes, la mort de Dieu - avec toutes ses conséquences, tous les troubles et les espoirs qu'elle annonce - est une de celles qui semblent le plus marquer la pensée, l'écriture et

1 G. Bataille, L'Expérience intérieure, [dans :] Idem, CEuvres complètes, Paris, Gallimard, 1973, t. 5, p. 121. 
l'existence même de Bataille. "Dieu est mort ! ", et c'est " nous [qui] l'avons tué ! ${ }^{2}$, s'exclame l'homme fou dans le Gai savoir, en acclamant par là même une nouvelle condition de l'homme et du monde. Cette constatation accorde ou même impose à l'homme une liberté, la liberté de la mise en doute de tous les systèmes qui l'assujettissent, celle de chercher ses valeurs à lui, d'être " au-delà du bien et du mal ". La vie, qui est la seule à avoir une valeur absolue parce qu'elle n'a d'autre but qu'elle-même, se voit affranchie de tout ce qui prétendait constituer son sens. Comme l'écrit Bataille, "la vie ne demeure entière que n'étant pas subordonnée à tel objet précis qui la dépasse. La totalité en ce sens a la liberté pour essence ${ }^{3}$. Mais, en même temps, l'acceptation de ce meurtre confronte I'homme avec "le néant et la disparition de l'âme ", avec le fait que " la pourriture attend le corps ", qu'il n'y a " plus de salut, de consolation, d'espoir $»^{4}$.

Aussi l'homme doit-il faire face au fait que Dieu est mort - de sa propre main - et cela le dépasse : " La grandeur de cet acte n'est-elle pas trop grande pour nous ? $\nu^{5}$. Qui alors pourrait être à sa hauteur ? Nietzsche écrit : "Ne nous faut-il pas devenir nous-mêmes des dieux pour apparaître seulement dignes de lui ? ${ }^{6}$. Mais par quelle voie I'homme peut-il devenir Dieu ? À la fin de son discours, le fou nietzschéen constate qu'il est encore trop tôt pour y arriver. Quant à Bataille, Michel Surya présente son point de vue ainsi : "Dire O u i au monde, lui dire $\mathrm{O}$ u i sans réserve et sans condition [...], c'est atteindre Dieu ; et l'ayant atteint, le tuer pour le devenir à son tour. Mais être

2 F. Nietzsche, Le Gai savoir, P. Wotling (trad.), Paris, GF Flammarion, 2007, p. 177.

3 G. Bataille, Sur Nietzsche, [dans :] Idem, CEuvres complètes, Paris, Gallimard, 1987, t. 6, p. 18.

4 P. Louvrier, Georges Bataille, la fascination du Mal, Paris, Rocher, 2008, p. 37.

${ }^{5}$ F. Nietzsche, Le Gai savoir, op. cit., p. 177.

${ }^{6}$ Ibidem. 
Dieu, c'est aussi, c'est surtout, devenir fou ${ }^{7}$. Peut-être seul l'homme "souverain ${ }^{8}$ est-il capable de le faire, peut-être seule " une nouvelle espèce de philosophes, qui soient dotés d'un goût et d'un penchant autres, inverses de ceux qui ont existé jusqu'à aujourd'hui - des philosophes du dangereux peut-être à tous les sens du terme ${ }^{9}{ }^{9}$ est-elle capable de l'imaginer.

Bataille, à plusieurs égards, s'avère proche de ce nouveau philosophe du dangereux imaginé par Nietzsche. Et cependant, c'est moins à la suite d'une influence, d'un choix résolu, que d'un impératif intérieur. La pensée et l'écriture de Bataille ne sauraient être limitées à un pendant à celles de Nietzsche et on aurait ici affaire plutôt à une coïncidence qu'à une simple inspiration unilatérale. Si Bataille, ébloui, s'est, à un moment, posé la question : " pourquoi envisager d'écrire, puisque ma pensée - toute ma pensée - avait été si pleinement, si admirablement exprimée ? ${ }^{10}$, il n'en reste pas moins que c'est toujours sa pensée et l'expression qu'il lui trouve n'appartient qu'à lui. En ce qui concerne la mort de Dieu, Bataille, fasciné tout au long de sa vie par les côtés bas de l'existence, par l'obscène, l'immonde, par ce qui déchire et trouble, reste ici fidèle à ses préférences. Ce qu'il explore d'une manière presque obsessionnelle, c'est l'incarnation la plus maté-

7 M. Surya, Georges Bataille, la mort à l'œuvre, Gallimard, Paris, 1992, p. 333.

${ }^{8}$ La souveraineté - notion réapparaissant tenacement dans plusieurs textes de Bataille - nous intéressera ici surtout comme un postulant soustendant les actes de certains personnages d'Histoire de l'œil et du Bleu du ciel ainsi que le déroulement de l'action même de ces deux récits. Nous ne pouvons donc que renvoyer à l'ensemble des analyses que Bataille a consacrées à cette question qui « participe à la fois du divin, du sacré, du risible ou de l'érotique, du répugnant et du funèbre " (G. Bataille, La Souveraineté, [dans :] Idem, CEuvres complètes, Paris, Gallimard, 1976, t. 8, p. 251).

9 F. Nietzsche, Par-delà bien et mal, P. Wotling (trad.), Paris, GF Flammarion, 2000, p. 49.

10 Cité d'après M. Surya, Georges Bataille, la mort à l'œuvre, op. cit., p. 618. 
rielle, ignoble de la mort de Dieu : son cadavre. Car effectivement, "Dieu est mort. Dieu demeure mort " ${ }^{11}$, mais il est, il demeure toujours, bien que sous la forme horrifique du cadavre. Et si sa présence marque à tel point l'univers bataillien - surtout dans les récits d'avant la Seconde Guerre mondiale, l'Histoire de l'œil et Le Bleu du ciel, qui constituent notre corpus - ce n'est pas qu'il soit mis en scène en réponse à la lecture de Nietzsche, mais parce qu'il tombe (cadavre, du lat. cadere - tomber ${ }^{12}$ ) sur cette scène d'une façon irrévocable. II semble que ce soit en luttant contre la gravitation de Dieu mort, en bataillant avec ses dépouilles religieuses et idéologiques, que Lord Auch cherche à conquérir son titre de noblesse et sa souveraineté.

\section{$C i-g \hat{\imath} t$}

La mort de Dieu devrait être libératrice ; si elle ne l'est pas immédiatement, c'est parce qu'il reste un obstacle majeur à surmonter : le cadavre divin. Ainsi, bien que la mort de Dieu soit le premier pas conduisant l'homme vers un changement radical, vers une existence plus pleine, son cadavre l'empêche toujours d'assumer sa liberté. Avant que l'homme puisse dire " oui " à tout, il devrait donc s'affranchir, non de Dieu, car il est déjà mort, mais justement de sa dépouille et de toutes ses émanations. II n'est pas étonnant qu'Histoire de l'œil - le premier récit publié de Bataille (1928) et en même temps son premier texte après la découverte de Nietzsche - témoigne d'une lutte violente contre tout ce que Dieu mort désigne : " on dirait que ce premier livre [...] n'a d'autre but que de transgresser, bousculer et niveler ${ }^{13}$. À côté de la lecture " psycha-

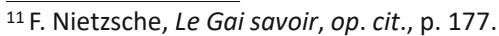

12 J. Kristeva, Pouvoirs de l'horreur, Essai sur l'abjection, Paris, Seuil, 1980, p. 11.

13 M. Leiris, " Du temps de Lord Auch », [dans :] Georges Bataille, Paris, Inculte, 2007, p. 182. 
nalytique "d'Histoire de l'œil, qui chercherait les traces de l'ombre du père de Bataille et qui viserait à démontrer que ce livre a été écrit " pour à la fois témoigner de son traumatisme et le surmonter ${ }^{14}$, une autre lecture s'impose : une lecture qui mettrait en relief la présence d'une autre ombre derrière cette œuvre, celle de Nietzsche, qui à côté de Sade " a le plus marqué Histoire de l'œil " $^{15}$, et avec ce philosophe, celle de la mort de Dieu qui constitue un traumatisme peut-être égal au traumatisme familial. Ainsi, Histoire de l'œil apparaît comme une des images les plus pleines, expressives du cadavre divin compris comme un obstacle, des serviteurs de celui-ci et, finalement, d'une bataille livrée à leur influence esclavagiste.

Histoire de l'œil a été publié sous le pseudonyme de Lord Auch. Comme le précise Michel Surya, "Lord : Dieu dans l'anglais des Écritures; Auch : abréviation triviale pour "aux chiottes" ${ }^{16}$. Cette signature peut renvoyer à la fois au nom du père - "Dieu se soulageant " $^{17}$ est le géniteur de Bataille, malade et "faisant sous lui "18 à la vue de son fils - et à celui du Père, puisque "le nom [Auch] est une façon abrégée de tout envoyer vers ce qu'en langage moins bas on appelle latrines ${ }^{19}$. Le nom étant précédé par le titre de Dieu, le sujet de ce traitement semble évident. Quant aux forces exécutives, elles apparaissent sous les traits de deux jeunes gens : le narrateur et son amie Simone. La deuxième est explicitement décrite comme un être " si avide de ce qui trouble les sens que le plus petit appel donne à son visage un caractère évoquant le sang, la terreur subite, le crime, tout ce qui

\footnotetext{
14 G. Ernst, "Histoire de l'œil, notice ", [dans :] G. Bataille, Romans et récits, Paris, Gallimard, 2004, p. 1002.

15 Ibidem, p. 1014.

${ }^{16}$ M. Surya, Georges Bataille, la mort à l'œuvre, op. cit., p. 115.

17 Ibidem, p. 116.

18 Ibidem.

${ }^{19}$ M. Leiris, " Du temps de Lord Auch », op. cit., p. 181.
} 
ruine sans fin la béatitude et la bonne conscience ${ }^{20}$. De son côté, répondant en quelque sorte à l'impératif nietzschéen : " y a-t-il chose plus belle que de partir à la recherche de ses propres vertus ${ }^{21}$, le narrateur constate : " j'achevai de perdre le sens de mots comme espoir et désespoir. J'éprouvai par lassitude une nécessité de donner malgré tout quelque sens à ma vie. Elle en aurait dans la mesure où je reconnaîtrais comme désirables un certain nombre d'événements » $(H C E, 12)$.

Ainsi, conformément à l'injonction qui découle du pseudonyme Lord Auch, le couple des héros principaux va conséquemment à l'encontre de tous les systèmes de valeurs traditionnels, des mœurs acceptées par la société, des autorités communément respectées, en un mot de tout ce qui reste après la mort de Dieu : de son cadavre. De jeux pervers mais encore plus puérils que périlleux, en passant par des manipulations qui provoquent la folie et finalement le suicide de leur " innocente amie " Marcelle, jusqu'au meurtre d'un jeune prêtre, c'est tout un cortège de débauches, de délires et de transgressions allant de plus en plus loin. L'attitude des deux adolescents n'est qu'une " manière dangereuse de résister aux sentiments de valeur habituels ${ }^{22}$. Déjà au début du récit, Simone, en urinant sur sa mère, tue, d'une manière symbolique, ce que cette vieille dame aux "yeux tristes " $(H C, 7)$ représente de traditionnellement respectable. De même, un peu plus tard, elle reprend ce geste meurtrier ${ }^{23}$ sur le cadavre de la "pure » Marcelle dont la pendaison « déjà [...] symbolise le suicide de Dieu ${ }^{24}$. On y sent déjà, comme l'écrit Gilles Ernst, " souffler le vent de Nietzsche [...] dans la révolte brutale contre les parents et leur peur du "scan-

${ }^{20} \mathrm{G}$. Bataille, Histoire de l'œil, [dans :] Idem, Romans et récits, op. cit., p. 4. Ensuite dans le corps du texte comme $H C$, suivi du numéro de la page.

${ }^{21} \mathrm{~F}$. Nietzsche, Par-delà bien et mal, op. cit., p. 187

22 Ibidem, p. 50.

${ }^{23}$ Cf. G. Ernst, « Histoire de l'œil, notice », op. cit., p. 1004.

24 Ibidem, p. 1007. 
dale", ou contre tous les vieillards, de corps ou d'esprit, qui pratiquent la morale de la "béatitude et de la bonne conscience" $»^{25}$. Mais, c'est l'épisode final du premier récit de Bataille qui apparaît comme le plus complexe dans la façon de présenter le cadavre divin et la lutte qui peut paraître paradoxale, parce qu'elle semble avoir pour but de tuer ce qui est déjà mort.

Après avoir quitté Madrid où Simone et le narrateur se sont réfugiés après la mort de Marcelle, ils se rendent en compagnie d'un Anglais débauché, Sir Edmond, à Séville, "ville de plaisir » $(H C E, 36)$. Là-bas, en visitant la cité, un jour, ils tombent sur une église où va s'accomplir, en quelque sorte, la campagne menée dès le début par le jeune couple. Cet épisode apparaît comme le point culminant de leurs aventures : toutes leurs fascinations, perversions, activités y sont reprises, amplifiées et poussées à l'extrême, c'est aussi leur essai final et le plus atroce dans l'entreprise du meurtre du cadavre divin. Ce qu'ils rencontrent sous le soleil andalou, c'est en effet le Dieu mort qui, de même que le corps se décomposant, se dissocie en plusieurs manifestations. D'abord, Don Juan : premier esclave et simultanément première émanation du défunt, ensuite la chair de l'église qui constitue en même temps et la tombe et le gisant, finalement, le prêtre, le dernier esclave et le dernier " martyr " de la croisade du narrateur et de son amie.

Ce qui suscite l'intérêt des héros et incite Simone à entrer dans l'église de la Caridad, c'est l'information que " celle-ci est l'église de Don Juan » $(H C E, 36)$. Il est pertinent de préciser à quel Don Juan ils ont affaire, car ce n'est pas celui de Mozart ou de Molière - débauché, libertin, se moquant de tout. Au contraire, Miguel Mañara y Vincentelo de Leca, fondateur de l'église de la Caridad, qui y est enterré, bien qu'il soit " un des modèles du Don Juan littéraire ", est un pécheur repenti, qui « après une jeu-

25 Ibidem, p. 1011. 
nesse qu'on dit dissipée [...] se marie en 1648 et devient un époux exemplaire " et qui " se fait moine après la mort de sa femme ${ }^{26}$. Ce Don Juan peut être interprété comme un exemple de l'homme souverain - au sens bataillien du terme - manqué : de l'homme qui a essayé de créer ses propres valeurs, de se construire, de dire " oui " à tout, de le dire surtout à la vie et à l'érotisme qui " est l'approbation de la vie jusque dans la mort ${ }^{27}$, mais qui y a finalement renoncé en choisissant des systèmes de valeurs tout prêts : les principes de la vie conjugale et l'ordre de la communauté religieuse. En tant qu'homme abandonnant sa liberté et se subordonnant à ce qui d'après Nietzsche devrait être rejeté ou du moins remis en question, il est non seulement un esclave, mais il constitue aussi une partie du corps divin, d'abord pour la simple raison qu'il est ce qui reste après la mort de Dieu, puis parce qu'il gît presque dans la maison de Dieu. Or, " que sont encore ces églises si ce ne sont pas les caveaux et les tombeaux de Dieu ? "28. C'est en tant que cette unité qu'il est soumis au traitement de Simone et de ses compagnons, car c'est à la fois son attitude de converti et ce à quoi il s'est converti que les héros attaquent dans la scène sur sa tombe.

Comment essayent-ils de tuer la morale, la foi, Dieu en Don Juan ? Moyennant deux instruments : le rire et l'urine. Simone, sortie de l'église,

riait aux éclats, ne pouvant parler. La contagion et le soleil aidant, je me pris à rire à mon tour, et même, à la fin, Sir Edmond.

«Bloody girl! s'écria l'Anglais, ne pouvez-vous expliquer ? Nous rions sur la tombe de Don Juan? "

Et riant de plus belle, il montra sous nos pieds une large plaque de cuivre ; elle recouvrait la tombe du fondateur de l'église [...].

Nos fous rires décuplés repartirent. Simone riant pissait le long des jambes : un filet d'urine coula sur la plaque. (HCE, 37)

26 Ibidem, p. 1031.

27 G. Bataille, L'Érotisme, [dans :] Idem, CEuvres complètes, Paris, Gallimard, 1987, t. 10, p. 17.

${ }^{28}$ F. Nietzsche, Le Gai savoir, op. cit., p. 178. 
Comme l'écrit Bataille, le rire poussé à l'extrême est " l'agonie de Dieu dans la nuit noire ${ }^{29}$. 11 touche au cadavre divin dans la mesure où il conteste Dieu et toute chose qui se veut l'instance qui donne un sens à la vie. Par ce rire Simone, comme Nietzsche, " proteste contre l'assignation d'un but aux choses, l'assignation d'un but au monde "; pour elle, de même que pour lui, "le monde n'a pas de but et par conséquent ce qu'il nous reste possible c'est de rire de ce qu'il est ${ }^{30}$. En riant à la sortie de l'église et sur la tombe de Don Juan, Simone atteste que leur Dieu n'est pas le but, le sens de la vie et qu'il n'est pas capable de les offrir. Ainsi, le fait de priver Dieu de sa raison d'être est le premier attentat contre le cadavre divin. Dans le second, Simone refait le geste qu'elle a exercé sur sa mère et sur Marcelle : elle urine. L'intention de ce geste est, elle aussi, identique : "Simone urine sur la tombe de Miguel Mañara comme elle a uriné sur sa mère, manière de marque son mépris pour le monde de la "béatitude et de la bonne conscience" ${ }^{31}$. Puis, tous les trois entrent dans l'église.

L'église - lieu sacré par excellence - qui est censée contenir symboliquement et réellement ce que le monde chrétien a de plus vénéré, estimable, qui devrait être un lieu d'échanges, de passages de biens spirituels, dans Histoire de l'œil apparaît comme un espace purement matériel, voire charnel. Le fait de la présenter de telle façon semble d'autant plus significatif que Bataille recourt rarement aux descriptions : " [il] n'est en effet pas un descripteur ", et dans Histoire de l'œil il n'y a " pas de pittoresque (sauf pour le retable de la Caridad) ${ }^{32}$. Le rapprochement de l'église et du corps commence par l'évocation du plafond qui « était fait d'une charpente ouvragée » (HCE, 37).

\footnotetext{
${ }^{29} \mathrm{G}$. Bataille, L'expérience intérieure, op. cit., p. 107.

${ }^{30} \mathrm{G}$. Bataille, Extrait d'un enregistrement radiophonique de 1959, cité d'après J.-P. Lorange, "Le rire de Zarathoustra », https://www.yumpu.com/ fr/document/view/3684291/le-rire-de-zarathoustra, p. 2-3.

${ }^{31} \mathrm{G}$. Ernst, " Histoire de l'œil, notice », op. cit., p. 1031.

32 Ibidem, p. 1021-1022
} 
La charpente est, bien sûr, un terme architectonique, mais c'est aussi, par extension, le squelette, les os. Ce mot est d'ailleurs repris un peu plus tard à l'occasion de la description des éléments décoratifs et de l'autel qui se trouvent au-dessous des poutres de la charpente. Là, la comparaison entre le corps et l'église est déjà établie d'une manière explicite : "Ce meuble de féerie, comme chargé des trésors de l'Inde, à force d'ornements, de volutes, de torsades, évoquait par ses ombres et l'éclat des ors les secrets parfumés d'un corps " (HCE, 37). Dans le passage qui suit, les visiteurs voient que ce qu'éclaire la lumière sanguine, charnelle, qui tombe " à travers les rideaux de cretonne rouge " $(H C E, 37)$, ce ne sont pas seulement les objets précieux et la chair "parfumée " de l'église : " à droite et à gauche de la porte, deux célèbres tableaux de Valdès Leal figuraient des cadavres en décomposition : dans l'orbite oculaire d'un évêque entrait un énorme rat... " $(H C$, , 37). Cet " ensemble sensuel et somptueux " avec " les jeux d'ombre et la lumière rouge de rideaux, la fraîcheur et l'odeur des lauriers-roses " (HCE, 37), étant donné la présence de ces tableaux, semble être marqué aussi par d'autres sensations, un peu moins somptueuses, et par une autre odeur, puisqu' " une anecdote veut que Murillo [dont Leal a été le disciple] ait dit qu'on se bouche le nez devant les toiles de Valdès Leal ${ }^{33}$. Qui plus est, le cadavre divin symbolisé par Don Juan est ici multiplié : sur une des toiles, à côté de l'évêque mentionné, figure justement le corps de "Miguel Mañara, revêtu du manteau de l'ordre de Calatrava ${ }^{34}$. La description de la maison de Dieu cède la place à l'apparition des esclaves suivants, parties du corps mort de celui-ci.

L'apparition du personnage central, le père Aminado, est annoncée par une pénitente. Elle le préfigure dans le sens où sa description touche déjà au champ sémantique

33 Ibidem, p. 1031.

34 Ibidem. 
lié à la mort, au cadavre, qui sera repris et développé dans le portrait du prêtre : " les traits pâles, extasiés : la tête en arrière, les yeux blancs, elle traversa la salle à pas lents, comme un spectre d'opéra " $(H C E, 38)$. Et, un moment après, le " confessionnal s'ouvrit. II en sortit un prêtre blond, jeune encore et très beau, les joues maigres et les yeux pâles d'un saint [...] le regard élevé vers un point du plafond : comme si quelque vision céleste allait l'arracher au sol » $(H C E, 38)$. Or, ce n'est pas une vision céleste qui va l'enlever, mais Simone. Celle-ci, ayant obtenu la confession, tient au prêtre un discours dévergondé, puis le moleste avec ses compagnons en l'arrachant ainsi à Dieu, puisque "la profanation de la confession par Simone et les sévices infligés à Aminado ont [...] le sens d'un adieu [...] à un passé révolu ${ }^{35}$. Ce passé révolu est le monde d'avant la mort de Dieu, monde dont Aminado reste un esclave cadavérique. Même avant sa mort, tout au long de la narration présentant ses "supplices ", revient un mot qui le désigne - la charogne : "Simone gifla la charogne sacerdotale. La charogne à ce coup rebanda " (HCE, 39), "Nous laissâmes tomber la charogne " (HCE, 41). Par cette dénomination et surtout sa condition de serviteur d'un " passé révolu ", Aminado appartient à la mort avant son décès réel.

Ernst voit en Aminado surtout le père de Bataille, mais il perçoit que le prêtre peut désigner aussi d'autres personnes : " n'était l'importance du traumatisme légué par le père réel, on dirait qu'en Aminado Bataille se libère d'abord de ces pères spirituels qu'il fréquente entre sa conversion de 1914 , et la fin de sa période catholique ${ }^{36}$. La deuxième hypothèse concernant la signification de l'Espagnol paraît être également justifiée. Alors, si on substitue au père de Bataille auquel Ernst pensait le Père des Évangiles et le père Aminado, il semble que les héros n'ont

35 Ibidem, p. 1007

36 Ibidem. 


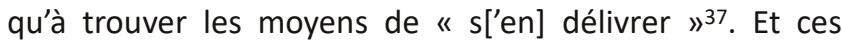
moyens passent, bien évidemment, par l'intermédiaire de la chair car " plus elle est sale, plus elle est souillée, plus tragiquement elle dit que Dieu n'est pas derrière qui promet une autre beauté, idéale celle-ci, imputrescible - que Dieu est mort. Et Dieu est mort d'autant plus que la chair est salie, d'autant plus qu'elle est souillée, d'autant plus qu'elle est belle ${ }^{38}$.

Ce processus de souiller la chair, en l'occurrence divine - car c'est celle d'Aminado et simultanément de Dieu dont il est serviteur et représentant sur Terre - commence par la confession "perverse " de Simone. Ensuite, il s'accélère : la soumission du prêtre aux différents actes sexuels, le fait que Simone urine comme avant (cette foisci sur les vêtements du prêtre), la profanation de l'hostie et du vin (dans la religion catholique du corps et du sang du Christ) commencée par le narrateur et ses amis mais aboutie par Aminado. Les excitations, les effrois, les espoirs du prêtre finissent avec un de ces derniers : " il murmura alors, la voix faible, mourante : "... le martyre..." Un espoir de salut venait au misérable " (HC, 41). Mais dans l'église, dans " ce grand monument didactique [...] qui courbait la foule entière ", qui, à un moment, " perdit le sens qui le fondait " et " se disloqua ${ }^{39}$, il n'y a plus de sens, de salut possibles. Pourtant, Aminado deviendra un martyre mais par les règles de Sir Edmond : "Tu sais que les pendus ou les garrottés bandent si fort, au moment de l'étranglement, qu'ils éjaculent. Tu seras donc martyrisé, mais en baisant " $(H C E, 41)$. Le prêtre assassiné d'une façon qui lie deux choses qu'il se refusait et auxquelles I'homme souverain va dire "oui ", à savoir la mort et l'érotisme, pour Simone est toujours trop peu " tué », trop peu « souillé ».

\section{Ibidem.}

${ }^{38}$ M. Surya, Georges Bataille, la mort à l'œuvre, op. cit., p. 44.

${ }^{39}$ G. Bataille, Manet, p. 127, cité d'après M. Surya, Georges Bataille, la mort à l'œuvre, op. cit., p. 569. 
II reste encore cette question d'Ernst, posée à propos d'Aristide Bataille, mais susceptible, encore une fois, d'expliquer le traitement auquel Aminado est assujetti aussi en tant que prêtre : " comment ne pas avoir ces "yeux châtrés" que le "Récit" attribue aux gens ne voulant pas voir l'“obscénité", sinon en tuant l'œil du père [...] ? ${ }^{40}$. Un des moyens de le faire et d' " essayer de se délivrer du père " est justement " introduire en soi un œil $»^{41}$, ce que fait Simone avec l'œil du cadavre sur lequel, un moment avant, "la mouche se déplaçait doucement " (HCE, 42). Est-ce que ce livre dont " le drame est celui de l'enfance de Bataille ; la vision est cet anti-idéalisme violent des années 1920, et la lecture est celle des écrivains dans cette même période (Nietzsche, Dostoïevski, Sade [...]) $»^{42}$ a libéré l'auteur du spectre paternel et du cadavre divin ? Gilles Ernst semble en douter : "la fin de "Récit" prouve que l'angoisse, l'œil de Marcelle enfoncé dans la vulve de Simone, n'a pas totalement disparu $~^{43}$, l'œil de Marcelle, du cadavre, du père, du prêtre, l'œil qui symbolise entre autres Dieu, l'œil du cadavre divin.

À moins que cet objet d'angoisse, de persécution, ne soit aussi l'objet de la plus haute voyance. Dans ce cas, l'épisode final d'Histoire de l'œil ne serait pas uniquement un acte violent visant le cadavre divin mais aussi une tentative de communication dans la mort, une tentative de restitution d' " un sacré actif, indiscuté, impérieux, dévorant ${ }^{44}$, à travers une voie sacrée de l'érotisme et du sacrifice/sacrilège. En effet, la mort et l'érotisme ayant le même fond sacré, ils coïncident, peut-être, de la manière la plus visible au moment de la transgression qu'introduit la fascination exercée par les deux. Car, bien que I'horreur

\footnotetext{
${ }_{40} \mathrm{G}$. Ernst, « Histoire de l'œil, notice », op. cit., p. 1007.

41 Ibidem.

42 Ibidem, p. 1001.

43 Ibidem, p. 1007.

${ }^{44}$ R. Caillois, L'homme et le sacré, Paris, Gallimard, 1950, p. 14.
} 
qu'inspire le cadavre - en l'occurrence le cadavre divin soit immense, l'attrait qu'il peut exercer se révèle parfois suffisant pour que l'abject devienne " cet obscur objet du désir ". Aussi Simone aurait-elle pu prendre pour devise ce propos de Louis-Vincent Thomas : "si l'orgasme est la "petite mort", c'est dans la vraie mort qu'il se réalise pleinement $"{ }^{45}$. De cette manière, Aminado est désirable justement en tant que cadavre, un être soumis au sacrifice profanateur, à une " action violente, privant la victime de son caractère limité et lui donnant l'illimité, l'infini qui appartient à la sphère sacrée ${ }^{46}$.

Ainsi, la mise à mort d'un serviteur de Dieu dans son temple serait en même temps la mise à mort de soimême, l'entrée dans le domaine de la souveraineté, de la mort. Le cadavre divin serait donc l'intermédiaire presque incontournable d'un élan sacrificiel plus global. Comme l'écrit Pierre Klossowski : " pour Bataille, le nom de $\mathrm{D}$ i e u est en quelque sorte la matière d'un co n tre-s acre m e $\mathrm{t}$, sur laquelle l'esprit n'agira que sur lui-même pour se détruire ${ }^{47}$. Dès lors, ce que l'abattage du cadavre divin devrait impliquer, c'est aussi la suppression du sujet. Klossowski précise encore ailleurs : " seule la vacance du moi répondant à la vacance de Dieu constituerait le moment souverain ${ }^{48}$. La vacance de ce moi dont Bataille implorait la disparition : " Dans le fond de la terre, / ô ma tombe, / délivre-moi de moi, / je ne veux plus l'être ${ }^{49}$.

\footnotetext{
${ }^{45}$ L.-V. Thomas, Les chairs de la mort, Corps, mort, Afrique, Paris, Institut d'édition Sanofi-Synthélabo, 2000, p. 483.

${ }^{46} \mathrm{G}$. Bataille, L'Érotisme, op. cit., p. 91.

47 P. Klossowski, "La messe de Georges Bataille ", [dans :] Idem, Un si funeste désir, Paris, Gallimard, 1963, p. 125.

48 P. Klossowski, "Le simulacre dans la communication de Georges Bataille ", [dans :] Critique, août-septembre 1963, nos 195-196, p. 750.

${ }^{49} \mathrm{G}$. Bataille, L'Expérience intérieure, op. cit., p. 72.
} 


\section{De mortels principes}

Cependant, afin de faire place à l'expérience athéologique telle que la comprend le théoricien et le praticien de la souveraineté, il faut encore nettoyer le champ de bataille - au moins de la bataille intérieure - des autres avatars du cadavre divin. Car Dieu n'est pas mort pour laisser la place à d'autres maîtres ou idéologies qui tenteraient de constituer le sens de la vie de l'homme, de lui montrer le but de cette vie et, finalement, de lui promettre un certain salut s'il allait se comporter d'une façon désirable : « Dieu mort, il n'y a plus de dieux possibles, plus de chef, plus de roi [...], plus de Führer pour qui vivre ou mourir... Rien de ce qui s'autorisa par le passé de Dieu, rien qui s'en justifia. Ce n'est ni la moindre ni la moins politique des conséquences de la mort de Dieu. II n'y a plus de sociétés monocéphales possibles ${ }^{25}$. Pas de sociétés monocéphales possibles - guidées par une seule tête, par un seul souverain qui briderait la souveraineté des autres, par une seule idéologie, par une seule croyance, d'autant plus que Dieu et le pouvoir centralisé en une seule personne - $a$ fortiori quand ce pouvoir tente de se légitimer par des valeurs supérieures - sont, dans une certaine mesure, interchangeables. Dans cette logique, l'attentat contre le pouvoir d'ici-bas a une dimension religieuse et celui contre Dieu un caractère politique. Pierre Klossowski, en analysant le geste révolutionnaire, constate que " l'exécution du Roi devient [...] le simulacre de la mise à mort de Dieu ${ }^{51}$. C'est d'ailleurs pourquoi Bataille, comme l'évoque Surya à propos des rites de la société secrète d'Acéphale, pratiquait " la commémoration de la décollation de Louis XVI sur la place de la Concorde, comme réaffirmant qu'y doit être "criée, hurlée, la mort de Dieu" au moment où ses

50 M. Surya, Georges Bataille, la mort à l'œuvre, op. cit., p. 292.

51 P. Klossowski, Sade mon prochain, précédé de Le philosophe scélérat, Paris, Seuil, 1967, p. 71. 
grimaces s'employaient à faire de son cadavre "une monstrueuse entreprise esclavagiste" ${ }^{52}$.

Pour Bataille, pendant la rédaction du Bleu du ciel, les grimaces de Dieu mort sont surtout le nazisme et le communisme ; le nazisme auquel il était très tôt hostile et le communisme dont il semble n'avoir jamais partagé les espoirs. Bien qu'il collabore avec le Cercle communiste démocratique et La Critique sociale animés par Boris Souvarine, " il ne partage ni l'analyse ni les fins $~^{53}$ des membres de ce milieu. Et même s'il faut encore attendre un peu pour que Bataille cesse complètement de s'engager politiquement, en 1935 - année de l'achèvement du Bleu du ciel - il fait déjà apparaître des personnages, des problèmes historico-politiques qui dévalorisent l'attitude de I'homme engagé qui croit aux idéaux d'un système proposé et qui cherche dans une idéologie le sens de sa vie et le salut possible. Comme le dit Jean-François Louette, le dessein de Bataille dans Le Bleu du ciel consiste à " établir la pathologie des illusions et des élans communistes et fascistes. Par rapport à Histoire de l'œil, on passe de la névrose individuelle à la névrose sociale ${ }^{54}$; il semble qu'on passe aussi du cadavre divin dans le sens religieux au cadavre divin surtout politique, les deux s'avérant également gênants pour Bataille, cet homme qui se veut souverain.

C'est ainsi que, dans Le Bleu du ciel - qui dans certaines parties se révèle comme " une vive satire de milieux d'extrême gauche " - il met en scène, sous les traits de Lazare, cadavre ressuscité, une figure importante du communisme dissident de l'époque que Bataille fréquente au Cercle communiste démocratique mais " avec qui il est cependant en désaccord politique» ${ }^{55}$. Comme l'explicite dans un brouillon de "lettre au Cercle » la philosophe en

52 M. Surya, Georges Bataille, la mort à l'œuvre, op. cit., p. 361.

53 Ibidem, p. 205.

54 J.-F. Louette, "Le Bleu du ciel, notice ", [dans :] G. Bataille, Romans et récits, op. cit., p. 1055.

55 Ibidem, p. 1038. 
question, Simone Weil, "la révolution est pour lui [Bataille] le triomphe de l'irrationnel, pour moi, du rationnel ; pour lui une catastrophe, pour moi, une action méthodique où il faut s'efforcer de limiter les dégâts ; pour lui la libération des instincts, et notamment de ceux qui sont couramment considérés comme pathologiques, pour moi, une moralité supérieure. Quoi de commun ? "56. Que Lazare - tout aussi bien dans sa description physique et caractérologique que dans les idées qu'elle représente et dans les propos qu'elle tient - puisse être considérée comme une image tant soit peu fidèle de Simone Weil est, c'est le moins qu'on puisse dire, discutable ${ }^{57}$. Or, ce qui nous intéresse ici est uniquement le personnage de Lazare et l'attitude qu'il semble représenter dans le récit.

En tant que partisane d'une cause vouée à l'échec, se subordonnant aux idéaux qui déterminent sa vie, cherchant le sens de cette vie dans l'activité politique et révolutionnaire et non dans l'existence elle-même, Lazare est, de fait, esclave et cadavre divin. Son nom souligne déjà le caractère cadavérique du personnage, car même s'il " pourrait évoquer la résurrection et sa liesse... II évoque

\footnotetext{
$\overline{56} \mathrm{~S}$. Weil, cité d'après S. Pétrement, La Vie de Simone Weil, avec des lettres et d'autres textes inédits de Simone Weil (nouvelle édition), Paris, Fayard, 1997, p. 306.

57 Sur la représentation de Simone Weil dans Le Bleu du ciel, ainsi que sur les relations entre la pensée de Bataille et celle de l'auteur de Réflexions sur les causes de la liberté et de l'oppression sociale, cf., entre autres : M. Surya, Georges Bataille, la mort à l'œuvre, op. cit., p. 262-263 ; S. Pétrement, La Vie de Simone Weil, op. cit., p. 308 ; B. Souvarine, "Prologue ", [dans :] La Critique sociale. Revue des idées et des livres, 1931-1934, réimpression : Paris, Éditions de la différence, 1983, p. 21 ; M. Esposito, "Une passion en commun. Extase et politique chez Georges Bataille et Simone Weil », [dans :] lignes, mai 2005, n 17, p. 172-192 ; S. Fraisse, " La représentation de Simone Weil dans Le Bleu du ciel de Georges Bataille ", [dans :] Cahiers Simone Weil, 1982, t. 5, n², p. 81-91. Cf. aussi la critique que Bataille a consacrée à L'Enracinement de Simone Weil et dans laquelle, pour décrire Simone Weil elle-même, il reprend les mots et les expressions qui ont déjà servi pour la description de Lazare : G. Bataille, "La victoire militaire et la banqueroute de la morale qui maudit » [dans :] Idem, CEuvres complètes, Paris, Gallimard, 1988, t. 11, p. 532-548.
} 
davantage avec Bataille le long séjour parmi les morts ${ }^{58}$. L'autre aspect du nom de Lazare qui fait d'elle le cadavre divin est que ce nom " exprime aussi le refus de la mort, au profit du salut ${ }^{59}$. L'homme souverain dira " oui » à la mort, à la vie et à la chair en rejetant les possibilités illusoires de n'importe quel salut, tandis que Lazare " rêve de révolution avec la même ferveur que les chrétiens de la résurrection. Avec elle le monde n'a plus de chair (sinon une chaire grise) : il n'est plus qu'une idée... une idée de salut ${ }^{60}$.

Lazare, par ses traits de caractère, sa physionomie, son allure est le cadavre divin en tant qu'individu, mais son nom désigne aussi une unité plus vaste. En effet « Lazare est l'autre monde de Bataille, ou, c'est selon, l'autre nom du monde qui fut le sien en 1934 : celui de la politique ${ }^{61}$. Dès la première description, elle est présentée d'une façon qui ne laisse pas de doutes sur son caractère funeste. "Sa silhouette décarcassée et noire [...] une stupide apparition du malheur "62 apparaît dans un bar-restaurant, pareille à un zombie : " elle avait l'air de ne rien voir devant elle, souvent elle basculait les tables en passant » $(B C, 125)$. Elle avait " la chair jaunâtre » $(B C, 125)$, "le teint de la peau un peu cadavérique » $(B C, 129)$, elle " répugnait physiquement " $(B C, 129)$, elle avait " une odeur de tombe » $(B C, 167)$,

tout en elle, sa démarche saccadée et somnambulique, le ton de sa voix, la faculté qu'elle avait de projeter autour d'elle une sorte de silence, son avidité de sacrifice contribuaient à donner l'impression d'un contrat qu'elle aurait accordé à la mort. $(B C, 134)$

Lazare est la mort, l'esclave, parce que, servant une cause perdue, "son agitation [est] stérile » $(B C, 125)$ et son

\footnotetext{
58 M. Surya, Georges Bataille, la mort à l'œuvre, op. cit., p. 257.

59 J.-F. Louette, "Le Bleu du ciel, notice », op. cit., p. 1079.

60 M. Surya, Georges Bataille, la mort à l'œuvre, op. cit., p. 257-258.

$61 \mathrm{lbidem}$.

62 G. Bataille, Le Bleu du ciel, [dans :] Idem, Romans et récits, op. cit., p. 25. Ensuite dans le corps du texte comme BC, suivi du numéro de la page.
} 
" sang pauvre de vierge sale " $(B C, 126)$ ne changera rien puisque, comme le conclut Jean-François Louette, "Lazare, qui ne connaît pas la convulsion sexuelle (en tant que "vierge sale") demeure, sur un plan politique, impuissante ${ }^{63}$.

Cette ascèse sexuelle est encore un élément qui la sépare de la souveraineté à la Bataille : esclave, elle rejette l'érotisme et la vie au profit des spéculations intellectuelles et de l'action "stérile ". Apparition d'un autre monde, elle ignore celui qui l'entoure, elle est comme " un esprit étranger à tout ; la maladie, la fatigue, le dénuement ou la mort ne comptaient pour rien à ses yeux " $(B C$, 125). Aussi n'évolue-t-elle pas, pas plus que ses convictions : elle " horrifiait par la constance de ses préoccupations " $(B C, 133)$, " elle donna le sentiment d'une conviction inébranlable " $(B C, 147)$. Bien qu'elle sache que " la classe ouvrière est foutue " $(B C, 147)$, elle reste fidèle à sa cause car " quoi qu'il arrive, nous devons être à côté des opprimés " $(B C, 147)$, et même si la défaite s'approche, " on peut toujours sauver son âme " $(B C, 147)$. Ici, Lazare révèle que, pour elle, le salut promis par le communisme n'est pas uniquement social mais aussi, ou peutêtre surtout, religieux. Elle montre aussi qu'elle agit par pitié - un sentiment qui d'après Nietzsche caractérise les esclaves, les faibles.

Même si Lazare, en gros, représente une figure méprisable pour l'homme souverain, ses relations avec le narrateur - Troppmann - sont loin d'être simples. Comme un cadavre, elle répugne et attire en même temps. Ses descriptions sont imprégnées de contradictions significatives: " elle mettait mal à l'aise : elle parlait lentement avec la sérénité d'un esprit étranger à tout [...]. Ce qu'elle supposait d'avance, chez les autres, était l'indifférence la plus calme. Elle exerçait une fascination " $(B C, 125)$. Tropp-

$\overline{63}$ J.-F. Louette, « Le Bleu $d u$ ciel, notice », op. cit., p. 1057-1058. 
mann - en se démenant entre les éléments de ce chiasme que Lazare tisse avec son entourage (gêne/sérénité, indifférence/fascination) - tantôt essaie de l'éviter, tantôt va chez elle ou cherche sa compagnie. D'un côté, il l'insulte, la traitant entre autres de " sale conne " $(B C, 167)$, " curé » $(B C, 167)$ ou " être abject » $(B C, 167)$, de l'autre, il lui confie ses secrets ou s'interroge: " je me demandai un instant si elle n'était pas l'être le plus humain que j'eusse jamais vu » $(B C, 181)$. D'une part, l'activité de Lazare est vaine et absurde pour le narrateur : " je ne prenais plus l'affaire au sérieux. Elle n'avait pas de sens à mes yeux " $(B C, 174)$; de l'autre, il dit : " j'avais peur de Lazare honteusement. Comme si j'avais eu des comptes à lui rendre » $(B C, 168)$ - comme s'il se sentait malgré tout obligé d'agir, comme s'il se sentait coupable de ne pas le faire. Tout de même, pour cet homme qui se veut libre, «le pis, évidemment, serait d'aboutir au moment où [il] n'agirai[t] plus selon [s]es passions, mais selon celles de Lazare " (BC, 174). Finalement, Troppmann ne s'engage pas dans la lutte à côté des communistes, pourtant il ne parvient pas non plus à se libérer d'images oppressantes dictées par "les grimaces " de Dieu mort. En effet, les problèmes que Troppmann essaie de contourner l'assiègent comme une sorte de délire, de maladie, même pendant son sommeil. Les deux rêves que Troppmann fait successivement à Paris et en Espagne recèlent ainsi une signification politique ${ }^{64}$ et ils peuvent être considérés, au moins en partie, comme la traduction des troubles d'un homme confronté à la mort de Dieu et à ses cadavres.

Dans le premier rêve, Troppmann se trouve dans un théâtre, avant " un spectacle si extraordinaire que l'attente [lui] donnait de l'angoisse " $(B C, 141)$. En effet, " l'attraction attendue " n'est rien d'autre que " l'apparition d'un vrai cadavre " $(B C, 141)$. Dans une salle décorée comme un lieu de funérailles - " un cercueil allongé au

${ }^{64}$ Cf. Ibidem, p. 1090. 
milieu du lit à baldaquin " $(B C, 141)$ - la bière s'ouvre " comme un rideau de théâtre ou comme un couvercle de boîte d'échecs " $(B C, 141)$ : la partie va-t-elle être jouée avec le cadavre attendu, la mort ou Le Mort même ? D'abord, tombe sur la scène un cadavre " à la fois inquiétant et séduisant " $(B C, 142)$. Cependant, même si " un tour cruel et plaisant venait d'être joué [...] la victime demeurait inconnue " $(B C, 142)$. Ensuite, le cadavre se transforme en un être géant pourvu d'un crâne de cheval " couvert d'un casque militaire " $(B C, 142)$, puis en Minerve cadavérique, s'agitant sur " un cimetière aux monuments de marbre blanc " $(B C, 142)$, qui laisse finalement la place à l'évocation de la statue du Commandeur. L'abondance du vocabulaire mortuaire ainsi que les descriptions de chaque apparition démontrent que le fil conducteur du rêve est effectivement le cadavre. Cependant, les figures qu'il incarne ont quelque chose de plus en commun, et ce n'est pas par hasard, par une simple association d'images, qu'elles apparaissent toutes sous des traits cadavériques. Comme le prêtre Aminado, comme Lazare, elles semblent revêtues des valeurs que la mort de Dieu dénonce.

Ainsi, derrière le " cadavre géant ", Louette reconnaît une réminiscence de l'expression - citée d'ailleurs par Simone Weil - de Rosa Luxemburg qui " qualifia la socialdémocratie allemande de "cadavre puant" ». Quant à Minerve, Louette rappelle que Susan Suleiman y " repère Lazare, c'est-à-dire [...] la Révolution menée sans espoir, vouant l'intellectuel à la mort ${ }^{65}$. Minerve, figurant la sagesse, est un cadavre aussi en tant que ratio stérile des philosophes et des systèmes critiqués par Nietzsche. Le Commandeur, qui obsède littéralement Troppmann, menace des Don Juan insoucieux. Face à ce défilé funèbre, Troppmann, pris d'angoisse, trouve la seule possibilité de sauvegarde dans le rire qui, de même que sur la tombe de Don Juan, est une manière de renverser Dieu, un moyen

65 Ibidem, p. 1090-1091. 
de devenir fou et, par là même, égal ou supérieur à la sagesse incarnée par Minerve.

Ce répertoire cauchemardesque trouve sa prolongation dans le rêve qui allie les sensations éprouvées en Espagne et les images de la Révolution française et de la Russie soviétique. Le dormeur, en se promenant dans une " construction de fer et de vitres, qui ressemblait à la vieille Galerie des Machines " $(B C, 181)$, découvre à chaque pas des débris et des rêves brisés des deux révolutions. Ainsi, la première partie du rêve est profondément marquée par le passé ; ce que Troppmann y retrouve sont " les souvenirs de la Révolution " $(B C, 181)$, " des scènes historiques" $(B C, 181)$, une image " presque effacée " $(B C, 182)$. Le cadavre qui y est enterré est la France d'avant, cette " momie [...] plus morte qu'aucune autre » $(B C, 182)$, comme si le degré de mort pouvait se décliner. Avec elle sont ensevelis aussi - pour reprendre l'expression de Baudelaire - " les immortels principes de 89 »66 $^{66}$ qui, comme tous les autres principes forgés par différentes idéologies, à l'heure de la mort de Dieu font eux-mêmes le démenti de leur prétendue immortalité. Après l'ancien régime, après la Révolution qui y met fin, c'est le temps d'un nouveau renversement. Dans une salle " plus oppressante » (BC, 181), Troppmann aperçoit des traces de la Révolution communiste dont l'image ressemble à celle de Lazare : « jamais je n'avais rien vu de plus crispant, rien de plus humain non plus " $(B C, 182)$. Cependant, même si "la passion révolutionnaire [lui] mont[e] lentement à la tête " $(B C, 182)$, elle semble aussi vaine que la passion dont les preuves gisaient dans la salle précédente.

En effet, comme "la momie française ", cette nouvelle image est enfermée dans un même décor symbolique de la tombe de Dieu : " l'espace vide était plus vaste et plus solennel que celui d'une cathédrale " $(B C, 181)$.

${ }^{66} \mathrm{Ch}$. Baudelaire, Le Spleen de Paris. Petits Poèmes en prose, R. Kopp (éd. critique), Paris, Gallimard, 2006, p. 202. 
Décor symbolique qui, à la fin, comme les cadavres qu'il recouvre, tombe en poussière - " j'entrai dans la grande nef vitrée, sachant que, d'un instant à l'autre, elle allait exploser " $(B C, 182)$, " il devient visible qu'il [l'édifice] explosait » $(B C, 183)$. Ainsi le temple de Dieu, ou des révolutions, ne laisse qu'une vision fuligineuse où " tout était irrémédiablement sombre et poussiéreux ॥ [BC, 183], comme si chaque révolution se débattait avec le cadavre de la précédente, pour être ensuite à son tour enterrée. Le rêve se clôt en " un tumulte suffocant, sans gloire, sans grandeur, qui se perdait en vain, à la tombée d'une nuit d'hiver " $(B C, 183)$ devant les enfants " excités ". La vision de ces enfants, qui " ne répondaient pas " $(B C, 183)$ à force d'être charmés par la violence, préfigure la fin du roman, d'autant plus que Troppmann, dès son réveil, a " une sensation de fuite, comme $s^{\prime}[i l]$ avai[t] passé la nuit en chemin de fer » $(B C, 183)$.

C'est effectivement durant un voyage en train en Allemagne que Troppmann rencontre un autre groupe d'enfants - cette fois-ci des Jeunesses hitlériennes - une autre image du cadavre et des esclaves divins. Pareils au groupe russe, "avec un visage de poupée " $(B C, 205)$, les enfants étaient « immobiles [...], en transe [...], envoûtés par le désir d'aller à la mort. Hallucinés par des champs illimités où, un jour, ils s'avanceraient, riant au soleil : ils laisseraient derrière eux les agonisants et les morts " $(B C, 205)$. Lazare était " l'oiseau de malheur " $(B C, 123)$, dans ses " présages sinistres " $(B C, 134)$ le narrateur voyait " une menace plus générale, suspendue au-dessus du monde » $(B C$, $124)$; la foule des jeunes Allemands est déjà une " marée montante du meurtre " $(B C, 205)$, le présage qui commence à s'accomplir. Devant cette " marée " Troppmann prend le train vers Paris. Jean-François Louette pose une question fondamentale quant à l'attitude de Troppmann (et de Bataille) envers tout ce que Lazare et les autres cadavres divins du récit représentent, en se demandant si, à la fin du récit, "Troppmann fuit devant le nazisme ou 
fuit la politique ». Si c'est le premier cas, " faut-il conclure et aller jusqu'à dire que Troppmann monte dans le train qui le conduit vers la Contre-attaque antifasciste? "Si c'est le deuxième cas, est-ce " une sortie hors du champ politique, et d'un mouvement qui s'amorce vers l'expérience "athéologique" (la méditation de l'absence de Dieu) ? "67 La méditation de l'absence de Dieu après les méditations sur ses " grimaces " serait peut-être finalement la libération annoncée par Nietzsche, la libération de Dieu, des chefs, des idéologies et des idéalismes, en un mot du cadavre divin. Il est possible que ce soit vers cela que va Troppmann.

\section{Les adieux}

Dans le cas de Bataille, la réponse est peut-être plus évidente. C'est Michel Surya qui situe « la fin en lui [en Bataille] de toute espérance historique, [...] de tout engagement historique ${ }^{68}$ un an après l'achèvement du roman, en 1936. C'est encore lui qui résume le mieux l'attitude postérieure de Bataille et le rôle de Nietzsche dans ce processus : " C'est certes Nietzsche qui a sorti Bataille de l'expérience religieuse de sa jeunesse, Nietzsche qui l'a soustrait au désir de claustration pour lui faire découvrir l'agitation du monde. Mais c'est Nietzsche encore et paradoxalement, qui, en 1939, l'a replongé dans une expérience religieuse d'un autre ordre, et qui lui a fait condamner l'agitation ${ }^{69}$. Et, même si Bataille dira plus tard : " c'est l'exercice positif de la liberté non la lutte négative contre une oppression particulière qui m'éleva au dessus de l'existence mutilée ${ }^{70}$, ce qui frappe pendant la lecture d'Histoire de l'œil ou du Bleu du ciel, c'est la

67 J.-F. Louette, "Le Bleu du ciel, notice », op. cit., p. 1063.

${ }_{68}$ M. Surya, Georges Bataille, la mort à l'œuvre, op. cit., p. 486.

69 Ibidem.

${ }^{70}$ G. Bataille, Sur Nietzsche, op. cit., p. 18. 
cruauté, la brutalité de la manière dont le cadavre divin (autant religieux qu'idéologique) a été traité dans ces textes.

Dans ces deux récits, Bataille dénonce en effet, par les traits cadavériques, ce qui, selon lui, empêche l'homme de devenir libre, ce qui fait qu'il ne pense pas arbitrairement, qu'il se contente d'accepter les solutions toutes prêtes, solutions qui d'ailleurs non seulement limitent la liberté de penser et d'agir, mais aussi ne sont pas efficaces, ne remplissent pas leur rôle. Le christianisme qui, en tant que religion, devrait donner l'accès au divin, au sacré, ne le fait pas ou le fait dans une mesure insuffisante. Le communisme, promettant un " paradis " social, s'avère vain, stérile. À défaut de pouvoir changer quoi que ce soit, ses partisans apparaissent chez Bataille comme des soi-disant messies, conscients de leur impuissance, luttant pour le salut qui se révèle moins social que religieux. Les nazis, de leur côté, s'agitent comme des cadavres ambulants aveuglés par la volonté de leur maître et semant la mort tout autour.

Aussi, pour Bataille, l'homme, après avoir tué Dieu, a dû encore dépasser en lui les illusions, les espérances proposées par ses avatars, ses cadavres. Néanmoins, dans cette lutte même, se dessine déjà une autre possibilité, même si c'est une possibilité de l'impossible. En effet, si Bataille taquine constamment le cadavre divin, c'est parce qu'il est, pour lui, non seulement détestable mais aussi désirable. Comme les cadavres en général, celui de Dieu attire et répugne en même temps, il est danger suprême et objet de culte par excellence. Ceci explique, au moins en partie, les sentiments ambigus que provoque Lazare et l'attitude simultanément méprisante et fascinée de Simone et du narrateur envers le prêtre. Ainsi, le sacrifice profanateur d'un côté, la révolution et la guerre qui se prépare, de l'autre, fonctionnent aussi comme le prélude à l'expérience souveraine. Ils introduisent à la mort : au déchirement du monde et du soi-même. 
Qu'il soit l'ennemi visé dans une campagne violente ou l'intermédiaire d'une expérience intérieure, le cadavre participe à une quête de sens. Que ce soit le sens proposé par le christianisme, le salut aperçu par certains dans le communisme ou le manque de sens rationnel, c'est le cadavre qui, entre autres, les révèle ici. Et puisqu' « il est permis de penser que la première expérience métaphysique de l'animal humain, indissolublement esthétique et religieuse, fut cette bouleversante énigme : le spectacle d'un individu passant à l'état d'anonyme gélatine " $^{71}$, le cadavre est un des moteurs de toutes les questions fondamentales que l'homme ne cesse de se poser depuis le moment où il est devenu ce qu'il est : un être conscient de sa mortalité. Et même si l'on peut essayer prudemment d'éviter de les poser - par exemple en se jetant dans l'activité - pour certains, tel Bataille, la fuite n'est réellement ni possible ni désirable. Que Bataille ait trouvé des réponses philosophiquement ou existentiellement plus justes ou probantes que celles d'une Simone Weil n'est pas évident, mais il a certainement su leur donner une forme, celle du cadavre, qui, sous ses multiples apparitions, est à la fois fascinante et répugnante.

${ }^{71} \mathrm{G}$. Bucher, La Vision et l'énigme. Éléments pour une analytique du logos, cité d'après R. Debray, Vie et mort de l'image. Une histoire du regard en Occident, Paris, Gallimard, 1992, p. 37. 


\section{bibliographie}

Bataille G., La Souveraineté, [dans :] Idem, CEuvres complètes, Paris, Gallimard, 1976, t. 8.

Bataille G., " La victoire militaire et la banqueroute de la morale qui maudit » [dans :] Idem, CEuvres complètes, Paris, Gallimard, 1988, t. 11.

Bataille G., Histoire de l'œil, [dans :] Idem, Romans et récits, Paris, Gallimard, 2004.

Bataille G., Le Bleu du ciel, [dans :] Idem, Romans et récits, Paris, Gallimard, 2004.

Bataille G., L'Érotisme, [dans :] Idem, CEuvres complètes, Paris, Gallimard, 1987, t. 10.

Bataille G., L'Expérience intérieure, [dans :] Idem, CEuvres complètes, Paris, Gallimard, 1973, t. 5.

Bataille G., Sur Nietzsche, [dans :] Idem, CEuvres complètes, Paris, Gallimard, 1987, t. 6.

Baudelaire Ch., Le Spleen de Paris. Petits Poèmes en prose, R. Kopp (éd. critique), Paris, Gallimard, 2006.

Caillois R., L'homme et le sacré, Paris, Gallimard, 1950.

Debray R., Vie et mort de l'image. Une histoire du regard en Occident, Paris, Gallimard, 1992.

Ernst G., "Histoire de l'œil, notice ", [dans :] G. Bataille, Romans et récits, Paris, Gallimard, 2004.

Esposito M., "Une passion en commun. Extase et politique chez Georges Bataille et Simone Weil », [dans :] lignes, mai 2005, no 17.

Fraisse S., "La représentation de Simone Weil dans Le Bleu du ciel de Georges Bataille ", [dans :] Cahiers Simone Weil, 1982, t. 5, nº 2.

Klossowski P., "La messe de Georges Bataille », [dans :] Idem, Un si funeste désir, Paris, Gallimard, 1963.

Klossowski P., "Le simulacre dans la communication de Georges Bataille ", [dans :] Critique, août-septembre 1963, nos 195-196.

Klossowski P., Sade mon prochain, précédé de Le philosophe scélérat, Paris, Seuil, 1967.

Kristeva J., Pouvoirs de l'horreur, Essai sur l'abjection, Paris, Seuil, 1980.

Leiris M., "Du temps de Lord Auch », [dans :] Georges Bataille, Paris, Inculte, 2007.

Lorange J.-P., " Le rire de Zarathoustra ", https://www.yumpu.com/fr/ document/view/3684291/le-rire-de-zarathoustra.

Louette J.-F., "Le Bleu du ciel, notice ", [dans :] G. Bataille, Romans et récits, Paris, Gallimard, 2004.

Louvrier P., Georges Bataille, la fascination du Mal, Paris, Rocher, 2008.

Nietzsche F., Le Gai savoir, P. Wotling (trad.), Paris, GF Flammarion, 2007.

Nietzsche F., Par-delà bien et mal, P. Wotling (trad.), Paris, GF Flammarion, 2000.

Pétrement S., La Vie de Simone Weil, avec des lettres et d'autres textes inédits de Simone Weil (nouvelle édition), Paris, Fayard, 1997.

Souvarine B., "Prologue ", [dans :] La Critique sociale. Revue des idées et des livres, 1931-1934, réimpression : Paris, Éditions de la différence, 1983. 
Surya M., Georges Bataille, la mort à l'œuvre, Gallimard, Paris, 1992.

Thomas L.-V., Les chairs de la mort, Corps, mort, Afrique, Paris, Institut d'édition Sanofi-Synthélabo, 2000.

\section{abstract}

Funeral Bataille: Lord Auch versus the divine cadaver

Nietzsche is one of those philosophers and thinkers - or even the philosopher, the thinker - who had the most inspiring influence upon Georges Bataille's way of thinking as well as his existential choices. Among many Nietzschean concepts that are tenaciously present in Bataille's work one - namely the Death of God - seems to be particularly persistent. This article aims to analyze some of representations of the dead God, the divine cadaver, in both religious and ideological sense in two of Bataille's novels written before World War II: Story of the Eye and Blue of Noon.

\section{keywords}

Georges Bataille, Friedrich Nietzsche, the Death of God, cadaver

\section{mots-clés}

Georges Bataille, Friedrich Nietzsche, la mort de Dieu, cadavre

\section{paulina tarasewicz}

Assistante à l'Institut de Philologie Romane de I'Université de Gdańsk, membre de l'Équipe de Recherche en Théorie Appliquée (ERTA) et du comité de rédaction des Cahiers ERTA, traductrice. Elle prépare sa thèse portant sur Colette Peignot (Laure).

ORCID : https://orcid.org/0000-0001-6108-3370 